Copyright by the Acoustical Society of America. Harne, R. L. (2012). Theoretical investigations of energy harvesting efficiency from structural vibrations using piezoelectric and electromagnetic oscillators. Journal of the Acoustical Society of America, 132(1), 162-172. doi: 10.1121/1.4725765

\title{
Theoretical investigations of energy harvesting efficiency from structural vibrations using piezoelectric and electromagnetic oscillators
}

\author{
Ryan L. Harne ${ }^{\text {a) }}$ \\ Department of Mechanical Engineering, Virginia Polytechnic Institute and State University, \\ 151 Durham Hall, Mail Code 0238, Blacksburg, Virginia 24061
}

(Received 15 December 2011; revised 11 April 2012; accepted 30 April 2012)

\begin{abstract}
Conversion of ambient vibrational energy into electric power has been the impetus of much modern research. The traditional analysis has focused on absolute electrical power output from the harvesting devices and efficiency defined as the convertibility of an infinite resource of vibration excitation into power. This perspective has limited extensibility when applying resonant harvesters to host resonant structures when the inertial influence of the harvester is more significant. Instead, this work pursues a fundamental understanding of the coupled dynamics of a main mass-spring-damper system to which an electromagnetic or piezoelectric mass-spring-damper is attached. The governing equations are derived, a metric of efficiency is presented, and analysis is undertaken. It is found that electromagnetic energy harvesting efficiency and maximum power output is limited by the strength of the coupling such that no split system resonances are induced for a given mass ratio. For piezoelectric harvesters, only the coupling strength and certain design requirements dictate maximum power and efficiency achievable. Since the harvesting circuitry must "follow" the split resonances as the piezoelectric harvesters become more massive, the optimum design of piezoelectric harvesters appears to be more involved than for electromagnetic devices. (C) 2012 Acoustical Society of America. [http://dx.doi.org/10.1121/1.4725765]
\end{abstract}

PACS number(s): 43.40.At, 43.20.Tb, 43.38.Ar [JJM]

Pages: 162-172

\section{INTRODUCTION}

Harvesting vibrational energy for conversion to electrical power has been an active field of research within the last decade. ${ }^{1-5}$ Studies have often assumed an infinite source of energy exists which is convertible into electric power, dependent primarily on the mechanism of electromechanical conversion and specific design parameters of the mass-spring harvester devices. This is evident in the use of base vibration as the source of excitation in energy harvesting models, the infinite resource of energy. ${ }^{6-11}$ Metrics of performance of the harvester devices are given in absolute terms, e.g., milliwatts, indicating that a harvester design excited by an arbitrary oscillating base should theoretically supply so much power per input base acceleration. Power density, often evaluated in milliwatts per cubic centimeter, is also utilized for crossplatform comparison. . $^{3,10,12}$

The assessment of energy harvesting output in absolute terms may not accurately reflect the capability for various harvesting platforms to convert the available energy into electrical power. Since the devices are regularly embodied as electromechanical mass-spring systems, textbook analysis of such dynamic vibration absorbers (DVAs) suggests that their addition to a host structure could have significant dynamic influence on the main system. ${ }^{13}$ Depending on the inertial difference between the harvester and main system, the effect may be dramatic.

In this light, new research has begun to consider energy harvesting vibration absorbers (EHVAs) capable of both

\footnotetext{
a) Author to whom correspondence should be addressed. Electronic address: rharne@vt.edu
}

suppressing structural motion while converting a portion of the absorbed energy into electrical power. References 14-16 consider this application for skyscraper tuned-mass-dampers where the available energy harvest could be significant and where the device itself would play a critical role in attenuating structural vibration due to wind or seismic excitation. Reference 17 presented a model and numerical simulation of collocated DVAs and piezoelectric devices for concurrent beam attenuation and energy harvesting. The simultaneous energy harvesting from and vibration attenuation of lightweight structural panels has also been explored with distributed EHVA devices. ${ }^{18}$ Similarly, energy harvesting vibration isolators (EHVIs) have been considered particularly for vehicle suspensions employing electromagnetic components. ${ }^{19,20}$ In contrast to other energy harvesting research assuming an infinite vibrational energy reservoir, these studies approach a point at which satisfaction of the vibration control problem and maximizing energy harvested are intertwined goals.

Modeling and analysis for these vibration control and energy harvesting studies has focused on the specific application. Thus, modeling of right-angle beam ${ }^{21}$ and dualbeam $^{22,23}$ systems have been specific to those studies and presented greater emphasis on maximizing the harvested power as opposed to vibration suppression of the purely elastic sub-structure. Reference 24 formulated dimensionless power equations for a dual-mass harvester concept but did not also consider the simultaneous vibration control problem. The present study aims to provide a general and dimensionless framework from which to consider the vibration control and energy harvesting objectives without preference to either goal. In an application, one goal may take priority over the other but at present there has not been an attempt to 
formulate a maximum bound on harvestable vibrational energy, as observed in Ref. 25.

This paper presents the governing equations for the vibration of an excited main mass-spring-damper system to which an electromagnetic or piezoelectric EHVA is attached. The EHVAs are thereafter connected to external circuitry modeled as a simple resistive load, the convention in much energy harvesting literature. ${ }^{8-10}$ The present analysis therefore represents the inclusion of electromechanical effects to textbook DVA analysis ${ }^{13}$ with the additional viewpoint of harvesting the absorbed energy as electrical power. As with the classical DVA analysis ${ }^{13}$ and the more recent dual-mass harvester modeling, ${ }^{24}$ the present formulation remains dimensionless for greatest extensibility.

Following presentation of the governing equations, an introductory energy harvesting efficiency is presented. The objective of this efficiency metric is to determine how either electromagnetic or piezoelectric EHVAs should be designed (e.g., lightweight or heavy; impedance matching or mis-matching, etc.) and not a critical comparison between the electromagnetic and piezoelectric platforms themselves. Weakly and strongly coupled harvesters are considered to determine how the objectives of (i) attenuating the main mass (or structural) vibration and (ii) energy harvesting are interrelated. The EHVAs are observed to be passive, electromechanical DVAs in a true sense. Depending on the electromechanical conversion mechanism and strength of the coupling, certain design parameters may be suggested to maximize electrical power output and serve as a means for passive vibration attenuation of the host system.

\section{SYSTEM GOVERNING EQUATIONS}

Each system studied is composed of an EHVA attached to a main mass-spring-damper excited by an harmonic force. For clarity of meaning, at times throughout this work the main mass will alternatively be referred to as the "host" mass or structure; the attached EHVA will alternatively be referred to as the harvester or oscillator.

\section{A. Electromagnetic EHVA}

Consider a main mass-spring-damper $\left(m_{1}, k_{1}\right.$, and $\left.c_{1}\right)$ to which an electromagnetic EHVA $\left(m_{2}, k_{2}\right.$, and $\left.c_{2}\right)$ is attached, Fig. 1. As the main mass, $m_{1}$, is excited by an harmonic force, $f(t)$, the main mass will oscillate, $x(t)$, thereafter exciting the attached EHVA, $y(t)$. The magnetic mass, $m_{2}$, vibrates through the axis of a wound coil-having inductance, $L$; resistance, $R_{e}$; and electromagnetic coupling, $T=B \ell$ which is the product of magnetic flux density, $B$, and the coil length, $\ell$-inducing a flow of current in the coil. The coil is attached to an external circuit composed of a load resistance, $R$, across which a voltage is measured, $v(t)$.

The governing equations for this two degree-of-freedom (2DOF) system are derived using Lagrange's equations and may be expressed as ${ }^{26}$

$$
\begin{gathered}
{\left[\begin{array}{ccc}
m_{1} & 0 & 0 \\
0 & m_{2} & 0 \\
0 & 0 & L
\end{array}\right]\left[\begin{array}{l}
\ddot{x}(t) \\
\ddot{y}(t) \\
\ddot{q}(t)
\end{array}\right]+\left[\begin{array}{ccc}
c_{1}+c_{2} & -c_{2} & 0 \\
-c_{2} & c_{2} & -T \\
0 & T & \tilde{R}
\end{array}\right]\left[\begin{array}{c}
\dot{x}(t) \\
\dot{y}(t) \\
\dot{q}(t)
\end{array}\right]} \\
+\left[\begin{array}{ccc}
k_{1}+k_{2} & -k_{2} & 0 \\
-k_{2} & k_{2} & 0 \\
0 & 0 & 0
\end{array}\right]\left[\begin{array}{l}
x(t) \\
y(t) \\
q(t)
\end{array}\right]=\left[\begin{array}{c}
f(t) \\
0 \\
0
\end{array}\right]
\end{gathered}
$$

where $q(t)$ is the electric charge induced in the coil, $\tilde{R}=R_{e}$ $+R$ is the sum of the coil and external load resistances and $\left({ }^{\circ}\right)$ denotes the time derivative. Assuming the excitation force is harmonic, $f(t)=F_{o} \exp (j \omega t)$, where $\omega$ is the circular excitation frequency and $j=\sqrt{-1}$, the governing equations may be rewritten as

$$
\left[\begin{array}{ccc}
k_{1}+k_{2}-\omega^{2} m_{1}+j \omega\left(c_{1}+c_{2}\right) & -k_{2}-j \omega c_{2} & 0 \\
-k_{2}-j \omega c_{2} & k_{2}-\omega^{2} m_{2}+j \omega c_{2} & -j \omega T \\
0 & j \omega T & -\omega^{2} L+j \omega \tilde{R}
\end{array}\right]\left[\begin{array}{c}
x(\omega) \\
y(\omega) \\
q(\omega)
\end{array}\right]=\left[\begin{array}{c}
F_{o} \\
0 \\
0
\end{array}\right] .
$$

Dimensionless constants are then defined

$$
\begin{aligned}
& \omega_{1}=\sqrt{k_{1} / m_{1}}, \quad \omega_{2}=\sqrt{k_{2} / m_{2}}, \quad \zeta_{1}=c_{1} / 2 m_{1} \omega_{1}, \quad \zeta_{2}=c_{2} / 2 m_{2} \omega_{2}, \\
& \mu=m_{2} / m_{1}, \quad \Theta=\omega_{2} / \omega_{1}, \quad r=\omega / \omega_{1}, \quad \omega_{e}=\tilde{R} / \omega_{1} L .
\end{aligned}
$$

Normalizing for a unit main mass such that $\omega_{1}^{2}=k_{1}$ allows the governing equations to be reformulated as follows:

$$
\left[\begin{array}{ccc}
1+\mu \Theta^{2}-r^{2}+j 2 r\left(\zeta_{1}+\zeta_{2} \mu \Theta\right) & -\mu \Theta^{2}-j 2 \zeta_{2} \mu \Theta r & 0 \\
-\mu \Theta^{2}-j 2 \zeta_{2} \mu \Theta r & \mu \Theta^{2}-\mu r^{2}+j 2 \zeta_{2} \mu \Theta r & -j \frac{T}{\omega_{1}} r \\
0 & j & -r^{2}+j \omega_{e} r
\end{array}\right]\left[\begin{array}{c}
x(r) \\
y(r) \\
q(r)
\end{array}\right]=\left[\begin{array}{c}
\frac{F_{o}}{\omega_{1}^{2}} \\
0 \\
0
\end{array}\right]
$$




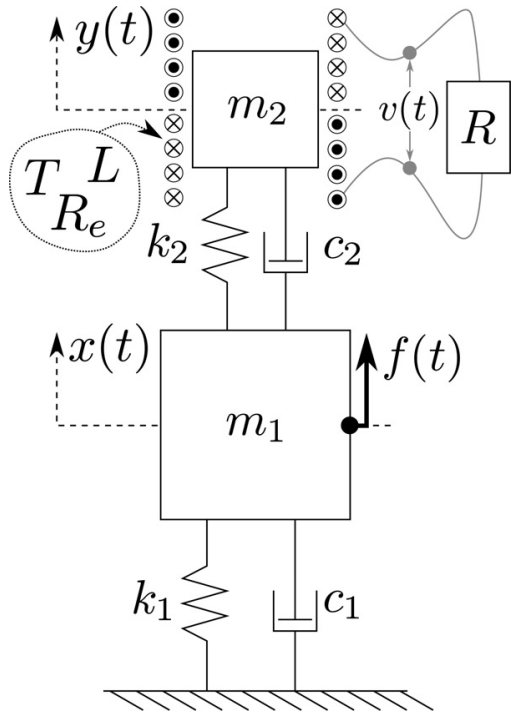

FIG. 1. Excited main mass with attached electromagnetic EHVA and external circuit.

Equation (4) shows that the effect of the electromagnetic induction is to dampen the EHVA vibration to varying degrees, dependent on adjustment of the external load resistance, $R$, and the strength of the electromagnetic coupling, $T$. The latter could be practically modified by the use of different magnetic masses (ferrite, rare-earth, and so on). The closed form solution to Eq. (4) is given in Appendix A. The induced current, $i(t)$, is related to the charge by $i=d q / d t$. The voltage across the external load resistance is then computed as $v(r)=R i(r)=R \dot{q}(r)=j \omega R q(r)$.

\section{B. Piezoelectric EHVA}

Figure 2 shows the coupled system with a piezoelectric spring EHVA. Electrodes are assumed to be attached to the top and bottom piezoelectric surfaces. The one-dimensional spring stiffness, $k_{2}$, may be computed from typical elastic and geometric parameters as $k_{2}=A Y / l$, where $A$ is the area of the electrodes (equivalent to the area of the top and bottom spring surfaces), $Y$ is the isotropic Young's modulus of the material and $l$ is the thickness of the piezoelectric spring. ${ }^{26}$ Throughout this analysis, $k_{2}$ will be used to follow existing convention as opposed to the higher-dimensional elastic parameters. The damping constant, $c_{2}$, could be evaluated from actual materials using experimental methods. ${ }^{27}$ The piezoelectric constant $d_{33}$ is used to characterize the

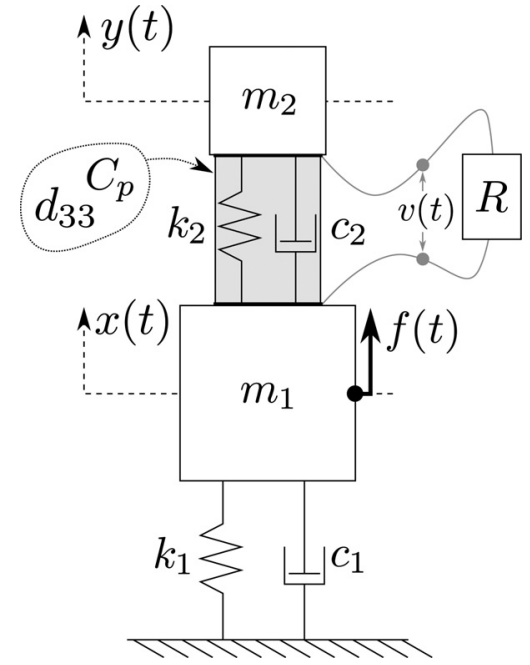

FIG. 2. Excited main mass with attached piezoelectric EHVA and external circuit.

strength of the electromechanical coupling, exhibited as an induced electrical field parallel to the poling direction (3) as the piezoelectric spring is deformed. The capacitance, $C_{p}$, may be computed from three-dimensional parameters as $C_{p}=A \epsilon^{T} / l$ where $\epsilon^{T}$ is the permittivity of the material calculated under constant stress.

Lagrange's equations are derived for the coupled electrodynamic response of the system

$$
\begin{aligned}
& {\left[\begin{array}{ccr}
m_{1} & 0 & 0 \\
0 & m_{2} & 0 \\
0 & 0 & 0
\end{array}\right]\left[\begin{array}{l}
\ddot{x}(t) \\
\ddot{y}(t) \\
\ddot{v}(t)
\end{array}\right]} \\
& +\left[\begin{array}{ccc}
c_{1}+c_{2} & -c_{2} & 0 \\
-c_{2} & c_{2} & 0 \\
d_{33} k_{2} & -d_{33} k_{2} & C_{p}\left(1-k^{2}\right)
\end{array}\right]\left[\begin{array}{c}
\dot{x}(t) \\
\dot{y}(t) \\
\dot{v}(t)
\end{array}\right] \\
& +\left[\begin{array}{ccc}
k_{1}+k_{2} & -k_{2} & -d_{33} k_{2} \\
-k_{2} & k_{2} & d_{33} k_{2} \\
0 & 0 & 1 / R
\end{array}\right]\left[\begin{array}{c}
x(t) \\
y(t) \\
v(t)
\end{array}\right]=\left[\begin{array}{c}
f(t) \\
0 \\
0
\end{array}\right],
\end{aligned}
$$

where $k$ is the electromechanical coupling factor calculated from $k^{2}=d_{33}^{2} Y / \epsilon^{T}$. Defining a normalized load resistance of $\omega_{p}=R C_{p} \omega_{1}$ and using the dimensionless parameters previously given in Eq. (3) allows the system in Eq. (5) to be reformulated as

$$
\left[\begin{array}{ccc}
1+\mu \Theta^{2}-r^{2}+j 2 r\left(\zeta_{1}+\zeta_{2} \mu \Theta\right) & -\mu \Theta^{2}-j 2 \zeta_{2} \mu \Theta r & -d_{33} \mu \Theta^{2} \\
-\mu \Theta^{2}-j 2 \zeta_{2} \mu \Theta r & \mu \Theta^{2}-\mu r^{2}+j 2 \zeta_{2} \mu \Theta r & d_{33} \mu \Theta^{2} \\
j \frac{d_{33} \omega_{1}^{2}}{C_{p}} \mu \Theta^{2} r & -j \frac{d_{33} \omega_{1}^{2}}{C_{p}} \mu \Theta^{2} r & \frac{1}{\omega_{p}}+j r\left(1-k^{2}\right)
\end{array}\right]\left[\begin{array}{c}
x(r) \\
y(r) \\
v(r)
\end{array}\right]=\left[\begin{array}{c}
\frac{F_{o}}{\omega_{1}^{2}} \\
0 \\
0
\end{array}\right] .
$$

In contrast to the electromagnetic coupling, piezoelectric effects modify the stiffness and the damping of the spring material. Thus, unlike the electromagnetic EHVA which is analogous to a variably damped DVA, the piezoelectric 
EHVA is analogous to a variably stiffened and damped DVA. The strength of the variation is related to the piezoelectric constant $d_{33}$ and the coupling factor $k^{2}$, both characteristic of certain piezoelectric materials. The closed form solution to Eq. (6) is given in Appendix B.

\section{ENERGY HARVESTING EFFICIENCY}

Several works have previously considered energy harvesting efficiency to be the induced electrical losses of the external circuit, which are dependent on the strength of the electromechanical coupling under consideration. ${ }^{28-30}$ This formulation assumes that the device is excited by a source of infinite energy (base vibration). When the excitation to the harvester is capable of being dynamically influenced, the definition of efficiency must be revisited. The present study considers that the harvester is excited by the main mass, but that the harvester may be inertially substantial to the point that it influences the natural frequencies of the fully coupled system. The coupling requires that the metric of efficiency be expressed in terms of the dynamics of the system components in question.

Fundamental principles may be used to arrive at an expression of efficiency. Let $E_{\text {in }}$ be the net energy input into the system by the exciting force and $E_{\text {out }}$ be the sum of losses experienced by the system. The first law of thermodynamics is employed:

$$
\begin{aligned}
& E_{\text {in }}=E_{\text {out }}, \\
& E_{\text {out }}=E_{m 1}+E_{m 2}+E_{e},
\end{aligned}
$$

where $E_{m 1}$ are the mechanical losses of the main mass, $E_{m 2}$ are the mechanical losses of the EHVA, and $E_{e}$ are the electrically induced losses. These energy losses may be computed by integration over a period of oscillation

$$
\begin{aligned}
E_{m 1} & =\int c_{1} \dot{x} \mathrm{~d} x \\
& =\int^{2 \pi / \omega} c_{1} \dot{x}^{2} \mathrm{~d} t \\
& =\pi \omega c_{1}|x|^{2} .
\end{aligned}
$$

Defining a change of variables, $z=x-y$, the losses $E_{m 2}$ may be computed:

$$
\begin{aligned}
E_{m 2} & =\int c_{2} \dot{z} \mathrm{~d} z \\
& =\int^{2 \pi / \omega} c_{2} \dot{z}^{2} \mathrm{~d} t \\
& =\pi \omega c_{2}|z|^{2} \\
& =\pi \omega c_{2}|x-y|^{2} .
\end{aligned}
$$

Whether considering the electromagnetic or the piezoelectric oscillators, the average power consumed by the load resistance $R$ is computed by $P(r)=|v(r)|^{2} / 2 R$. Integrating this over a period defines the electrical energy losses. Since the power is an average quantity, electrical losses are computed as the product of electric power and a period of oscillation:
TABLE I. System parameters used in electromagnetic studies.

\begin{tabular}{lcccc}
\hline \hline Strength & $k_{1}, \mathrm{~N} / \mathrm{m}^{2}$ & $R_{e}, \Omega$ & $L, \mathrm{H}$ & $T, \mathrm{~T} \cdot \mathrm{m}$ \\
\hline Weak & $1 e 6$ & 100 & 0.001 & 0.1 \\
Strong & $1 e 6$ & 100 & 0.001 & 1.0 \\
\hline \hline
\end{tabular}

$$
E_{e}=\frac{2 \pi}{\omega} \frac{|v|^{2}}{2 R}=\frac{\pi}{\omega} \frac{|v|^{2}}{R} .
$$

The energy harvesting efficiency of the EHVA is determined to be

$$
\begin{aligned}
\eta & =E_{e} /\left(E_{m 1}+E_{m 2}+E_{e}\right) \\
& =\frac{\pi|v|^{2} / \omega R}{\pi \omega\left(c_{1}|x|^{2}+c_{2}|x-y|^{2}\right)+\pi|v|^{2} / \omega R} \\
& =\frac{|v|^{2} / \omega_{1}^{3} R}{2 r^{2}\left(\zeta_{1}|x|^{2}+\zeta_{2} \mu \Theta|x-y|^{2}\right)+|v|^{2} / \omega_{1}^{3} R} .
\end{aligned}
$$

Equation (12) shows that like past work ${ }^{28,29}$ maximum efficiency is achieved in the absence of mechanical damping. However, even in the presence of minor mechanical damping, the interaction between the main system and oscillator plays a substantial role in modifying the efficiency of electrical conversion. This is because when the host mass is excited around resonance and assuming the tuning ratio is unity $\left(\Theta=\frac{\omega_{2}}{\omega_{1}}=1\right)$, the denominator component $|x-y|$ may be substantial given that the EHVA and host mass vibrate nearly out-of-phase.

Furthermore, in the latter event there is substantial deformation of the harvester spring which is the source of piezoelectric effects. Thus, even as the piezoelectric EHVA becomes more massive relative to the main mass, the harvester should be continually able to increase its efficiency. For electromagnetic EHVAs, the amplitude $|y|$ determines the resulting electrical output and it is not immediately evident from Eq. (12) how this factor influences the overall efficiency.

\section{HARVESTING PLATFORM PERFORMANCE}

Despite the use of dimensionless parameters, it is observed from Eqs. (4) and (6) that to determine the effects of electromagnetic and piezoelectric EHVAs, one must select parameters related to the electromechanical coupling strength, $T$ or $d_{33}$ and $k^{2}$, and related to the design of the harvester itself, $L$ and $R_{e}$ or $C_{p}$. Tables I and II provide the parameters used in simulation for the electromagnetic and

TABLE II. System parameters used in piezoelectric studies.

\begin{tabular}{lcccc}
\hline \hline Strength & $k_{1}, \mathrm{~N} / \mathrm{m}^{2}$ & $C_{p}, \mathrm{~F}$ & $d_{33}, \mathrm{~m} / \mathrm{V}$ & $k$ \\
\hline Weak & $5 e 11$ & $5 e-8$ & $-23 e-12$ & 0.12 \\
Strong & $5 e 11$ & $5 e-8$ & $-500 e-12$ & 0.56 \\
\hline \hline
\end{tabular}


(a) Vibration attenuation, $\Delta \mathrm{x}, \mathrm{dB}$

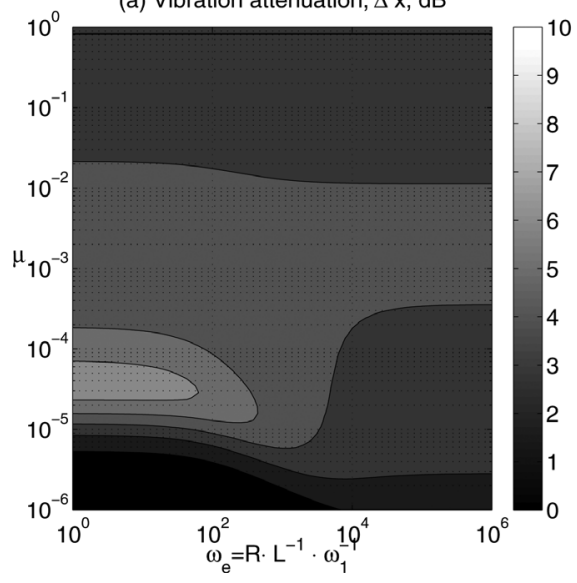

(b) $\log _{10}$ [maximum Power TF], W. $\left(\omega_{n}^{2} / F_{0}\right)^{2}$

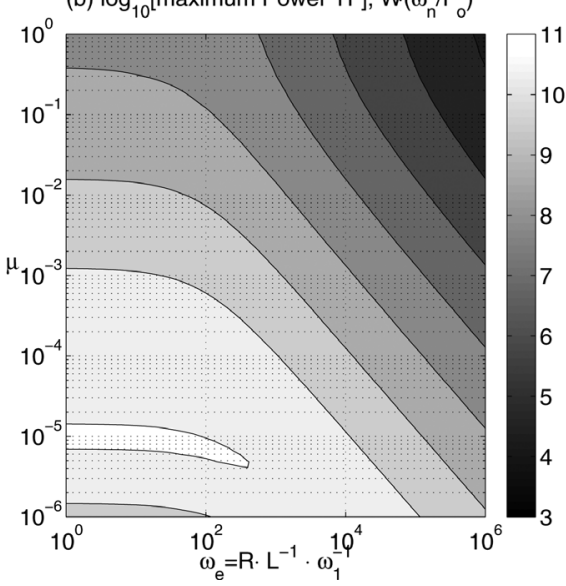

(c) Maximum efficiency

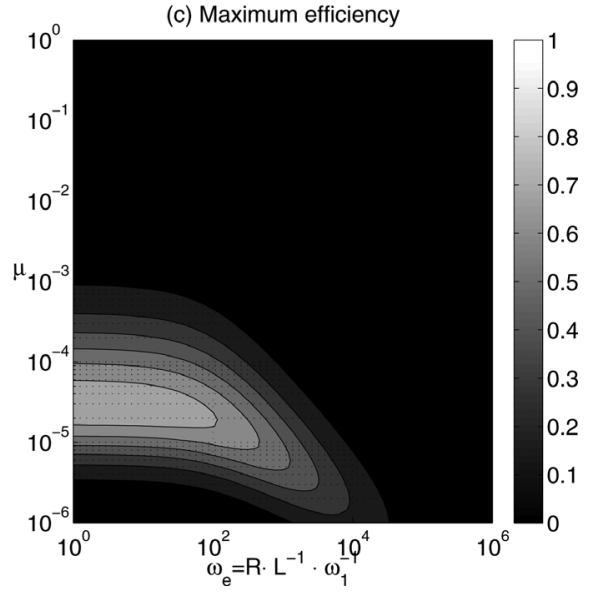

FIG. 3. System responses with weak electromagnetic coupling: (a) main mass vibration attenuation; (b) maximum power TF; (c) maximum efficiency.

piezoelectric studies, respectively. For each form of electromechanical coupling, a study is carried out for weak coupling and for strong levels of coupling. In declaring "weak" or "strong" electromechanical coupling, it must be understood that such designation is relative to the size of mass under consideration, since, for example, a weak coupling could still substantially affect the dynamics of a very lightweight oscillator. In all studies, the EHVA natural frequency is assumed to be identical to that of the primary system, $\Theta=1$; the magnitude of the excitation is unity, $F_{o}=1 \mathrm{~N}$; and the damping ratios are $\zeta_{1}=\zeta_{2}=5 e-4$.

The equations of Eq. (4) or (6) are solved over a range of $r(0.1<r<1.9)$ for selected values of $\mu$ and $\omega_{e}$. The metrics of present interest are the main mass vibration suppression, electrical power transfer function (TF) and the efficiency. To evaluate vibration attenuation, the convention of computing the $\mathrm{H}_{2}$ norm of the primary system is used. References 31 and 32 show that for linear DVA analysis, this is equal to the RMS main system response, $\sqrt{\left\langle x^{2}\right\rangle}$, normalized by the uniform power spectrum density, $S_{f}$, of the forcing excitation. In the present study, this metric is normalized by the RMS response of the main system with no EHVA and converted to a decibel scale. Thus, the attenuation of the main system is computed as

$$
\Delta x=-20 \log _{10} \frac{\sqrt{\frac{\langle x\rangle_{\text {with EHVA }}}{2 \pi S_{f} \omega_{1} / k_{1}^{2}}}}{\sqrt{\frac{\langle x\rangle_{\text {no EHVA }}}{2 \pi S_{f} \omega_{1} / k_{1}^{2}}}}=-10 \log _{10} \frac{\langle x\rangle_{\text {with EHVA }}}{\langle x\rangle_{\text {no EHVA }}} .
$$

The electrical power TF, $P(r)$ having units $W\left(\omega_{1}^{2} / F_{o}\right)^{2}$ $=W / N^{2} s^{4}$, and efficiency are also affected by the frequency shifting caused by the dynamic coupling and therefore maxima are computed as well as the specific value resulting at the EHVA natural frequency, $\omega_{2}$. Maximal electrical power and efficiency values would indicate the external power harvesting circuitry is capable of adjusting its switching frequency (converting ac to dc) for optimal performance while values computed for $\omega_{2}$ would indicate the external circuit is static and only harvests power at the original tuned frequency of the harvester.

\section{A. Electromagnetic, weak coupling}

Figure 3 plots the system responses of (a) main mass vibration attenuation, (b) maximum power TF, and (c) maximum efficiency when the weakly coupled electromagnetic (a) Vibration attenuation, $\Delta \mathrm{x}, \mathrm{dB}$

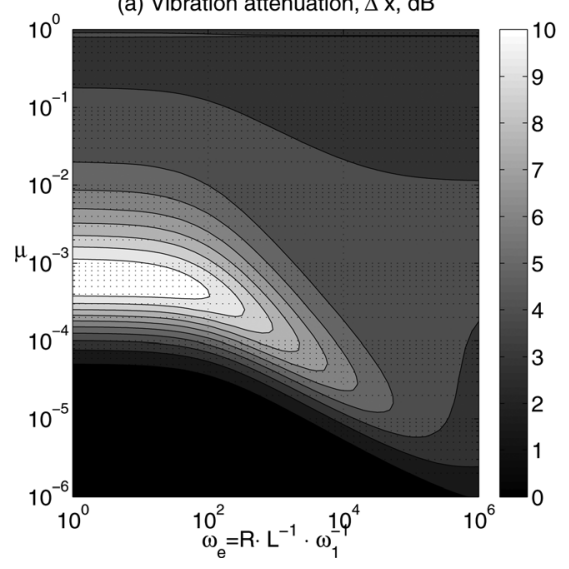

(b) $\log _{10}$ [maximum Power TF], W. $\left(\omega_{n}^{2} / F_{0}\right)^{2}$

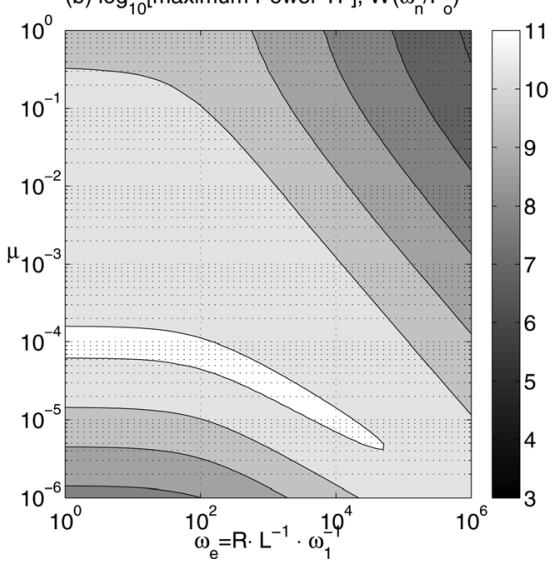

(c) Maximum efficiency

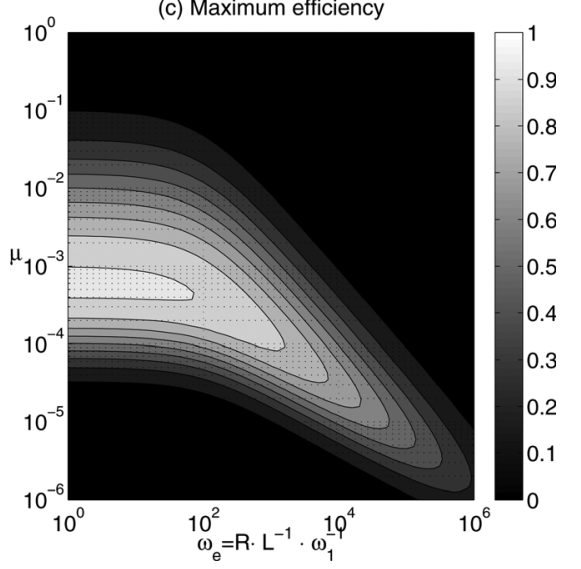

FIG. 4. System responses with strong electromagnetic coupling: (a) main mass vibration attenuation; (b) maximum power TF; (c) maximum efficiency. 


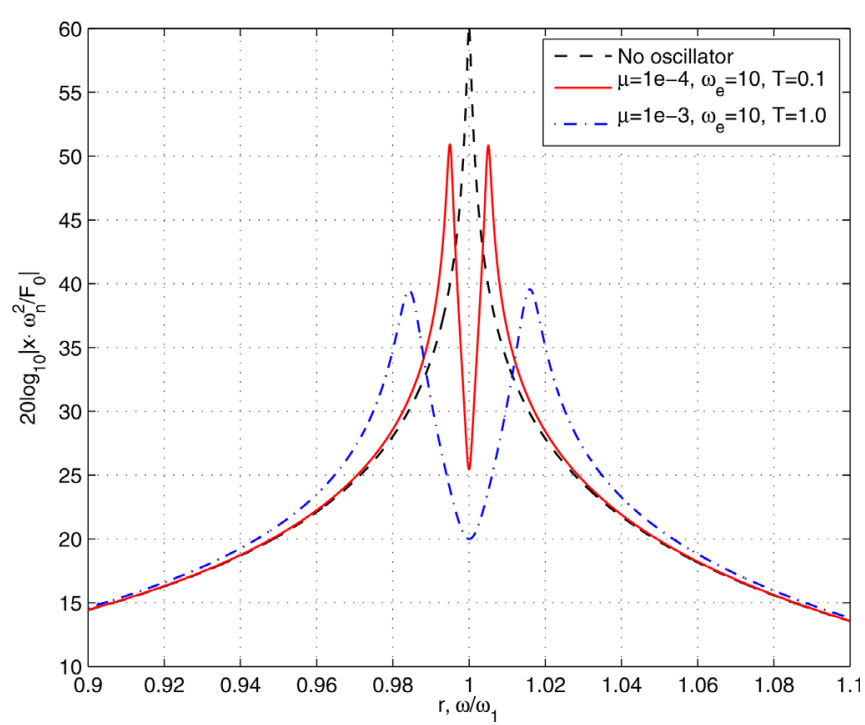

FIG. 5. (Color online) Main mass vibration frequency response function with weak and strong electromagnetically coupled oscillators.

EHVA is attached to the excited main mass. Contour lines for this and subsequent figures represent $1 \mathrm{~dB}$ increments for vibration attenuation; one order of magnitude for the power TF; and one-tenth increments for harvesting efficiency. Figure 3(a) shows that greatest vibration suppression is achieved for $1 e-5<\mu<1 e-4$ and $\omega_{e}<100$. This normalized frequency, $\omega_{e}$, corresponds to the condition that $R \leq R_{e}$, the load resistance being no greater than the coil resistance. Thus, impedance matching the external circuit to the EHVA electrical characteristics maximizes vibration attenuation.

Recall from textbook analysis that the addition of the DVA will "split" the original main system resonance into two resonance peaks above and below the original. ${ }^{13}$ Analysis shows that maximum suppression of these peaks occurs for the choice of DVA damping ratio which equalizes the amplitude of the split resonances. In observing the governing equations, the coupling strength determines the additional induced damping in the EHVA while the mass ratio determines the dynamic influence upon the main system. For the specific strength of electromagnetic coupling used in this scenario, $T=0.1 \mathrm{Tm}$, the EHVA with $\mu$ around $1 e-4$ is damped such that its influence on the host structure is to equally damp the two split resonances and provide maximum attenuation. (This feature will be further explained in Sec. IV B and Fig. 5.)

Maximum energy harvesting, Fig. 3(b), is seen to be achieved on the periphery of the optimum selection of $\mu$ which best serves to attenuate vibration. Thus, the optimally damped "vibration absorber" is not necessarily the equivalent to the optimally designed "energy harvester" for electromagnetic systems. Interestingly, the maximum harvesting efficiency spans this parameter divide between optimum vibration attenuation (for low $\mu$ ) and optimum energy harvesting, suggesting an important connection may exist between the two objectives.

\section{B. Electromagnetic, strong coupling}

Figure 4 plots the results computed for the case of strong electromagnetic coupling. A more substantial increase in main mass vibration attenuation is observed, Fig. 4(a), for the use of a mass ratio $\mu \approx 0.001$ with $\omega_{e}<100$. This result is also explained by the generation of optimal damping of the split resonances occurring due to the vibration absorber effects.

As for the weak coupling case, maximum electrical power is achieved for a selection of $\mu$ less than that which maximizes vibration attenuation but the same region of $\omega_{e}$, Fig. 4(b). Likewise, it is found that greatest efficiency appears for a choice of mass ratio $\mu$ in between the optima for the vibration control and energy harvesting objectives.

Note that low $\omega_{e}$ indicate a low value of external harvester circuit resistance $R$. Since the coil resistance is $R_{e}=100 \Omega$, results for both Figs. 3 and 4 show that any increase of $R$ above $R_{e}$, i.e., $\omega_{e}>100$ in the simulations, reduces the mechanical and energy harvesting performance of the EHVA. As earlier explained for best vibration attenuation, impedance matching the external circuit to the harvester electrical characteristics maximizes energy harvesting performance.

Finally, Fig. 5 plots the main mass vibration TF for the cases which best attenuate the main mass vibration as described above and when no oscillator is attached. It is (a) $\log _{10}\left[\right.$ Power TF at $\left.\omega_{2}\right], W \cdot\left(\omega_{n}^{2} / F_{o}\right)^{2}$

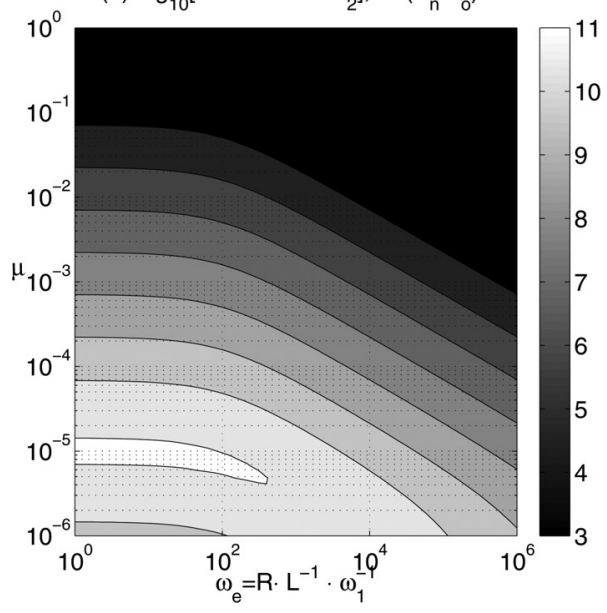

(b) Efficiency at $\omega_{2}$

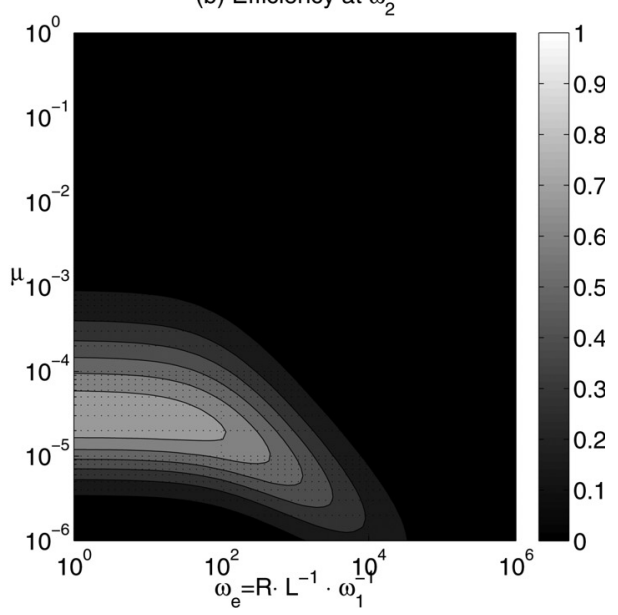

FIG. 6. (a) Power TF of weak electromagnetic oscillator at resonance $\omega_{2}$ and (b) efficiency at resonance. 
(a) $\log _{10}\left[\right.$ Power TF at $\left.\omega_{2}\right], W \cdot\left(\omega_{n}^{2} / F_{o}\right)^{2}$

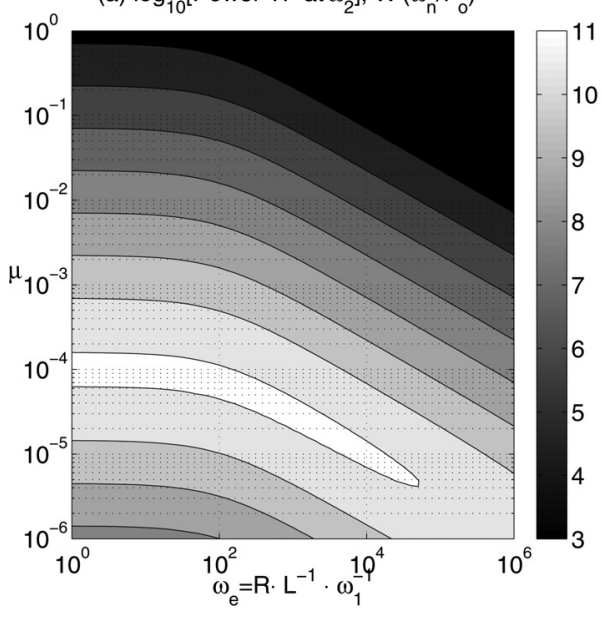

(b) Efficiency at $\omega_{2}$

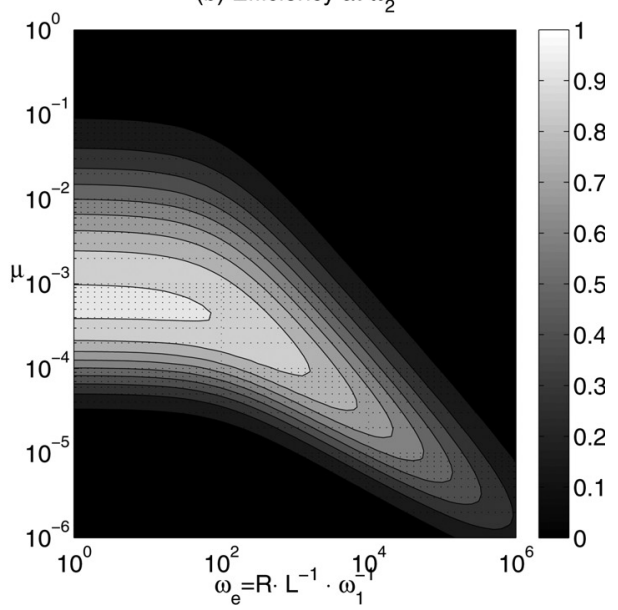

FIG. 7. (a) Power TF of strong electromagnetic oscillator at resonance $\omega_{2}$ and (b) efficiency at resonance. observed that for a certain choice of coupling strength $T$ the modification of mass ratio $\mu$ thereafter serves to yield split resonances of equal amplitude and damped to a maximum degree. This is a classic example of optimal damping for a DVA design ${ }^{13}$ although now achieved through electromechanical parameters and not mechanical damping adjustment.

\section{Electromagnetic harvesting at resonance}

Figures 6 and 7 plot the electromagnetic oscillator power TF and efficiency computed at the device resonance, $\omega_{2}$, as opposed to following the maximum values due to the generation of split resonances. Both figures show that best energy harvesting and efficiency occur for the same selection of $\mu$ and $\omega_{e}$ as those which followed the split resonances. Selection of $\mu$ above or below these optimal values show uniform decreases in device energy harvesting performance.

A question arises as to why the results of Figs. 6(a) and 7(a) are different than those observed in Figs. 3(b) and 4(b). The dynamic coupling between the two masses explains this effect. Figure 8 plots the power TF for $\omega_{e}=10$ and $T=$ $0.1 \mathrm{~T} \mathrm{~m}$ as the mass ratio is increased. For $\mu=1 e-6$, the res-

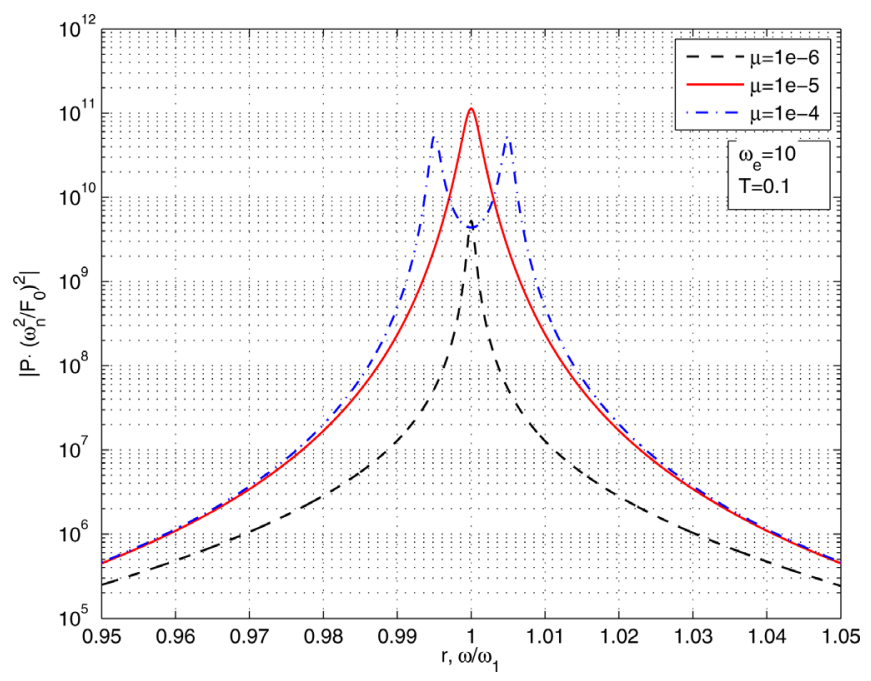

FIG. 8. (Color online) Power TF variation as mass ratio is increased, weak electromagnetic coupling. onance is sharp and precisely at $r=1$ due to the tuning ratio of unity, $\Theta=1$; thus, maximum efficiency and harvested power both occur at the EHVA natural frequency, $\omega_{2}$. For $\mu=1 e-5$, the resonance becomes somewhat more damped but best harvesting is still achieved at $\omega_{2}$. For $\mu=1 e-4 \mathrm{a}$ split in the resonance of the power TF occurs which corresponds to the oscillator becoming more inertially influential to the main mass vibration yielding two distinct resonances at $r=0.995$ and $r=1.005$. Therefore, maximum power $\mathrm{TF}$ and efficiency shift away from $\omega_{2}$ and occur at the location of the split resonances. Thus, it is found that for electromagnetic EHVAs, maximum power TF and efficiency occur at the harvester natural frequency (when $\Theta=\omega_{2} / \omega_{1}=1$ ) for the highest mass ratio $\mu$ such that the electromagnetic coupling strength is substantial enough to prevent the coupled system resonance from splitting.

\section{Piezoelectric, weak coupling}

The analyses were repeated with the piezoelectric governing equations using the parameters in Table II. Results of main mass vibration attenuation, maximum power $\mathrm{TF}$ and maximum efficiency are provided in Fig. 9 for the weakly coupled piezoelectric EHVA as $\mu$ and $\omega_{p}$ are modified. Maximum vibration suppression is achieved when $\omega_{p}=1$ and for $\mu \geq 0.001$. However, as compared with electromagnetic EHVAs, there is a less obvious optimal choice of parameters for attenuation performance. This is primarily due to the orders of magnitude in difference between the mechanical and electrical coefficients in the electrical equation of Eq. (4) for piezoelectric EHVAs. For electromagnetic EHVAs, these coefficients are nearly of the same magnitude, inducing far greater electromechanical damping per mass and providing a well-defined parameter selection for maximum vibration attenuation.

For the weakly coupled piezoelectric EHVAs, maximum electrical power is achieved for the most inertially substantial harvesters. This is explained by the coupling mechanism itself: piezoelectric charge generation is due to strain. Greater spring deformation, which would be the result of a larger (heavier) oscillator mass, would yield increased electrical power output. Likewise, the maximum harvesting 
(a) Vibration attenuation, $\Delta x, d B$

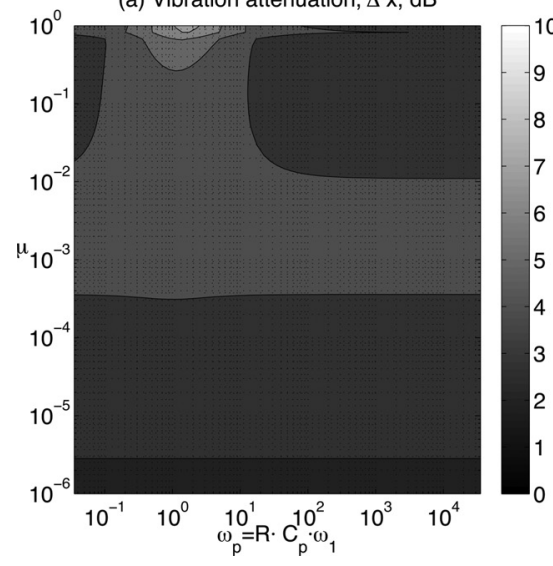

(b) $\log _{10}$ [maximum Power TF], W $\left(\omega_{n}^{2} / F_{0}\right)^{2}$

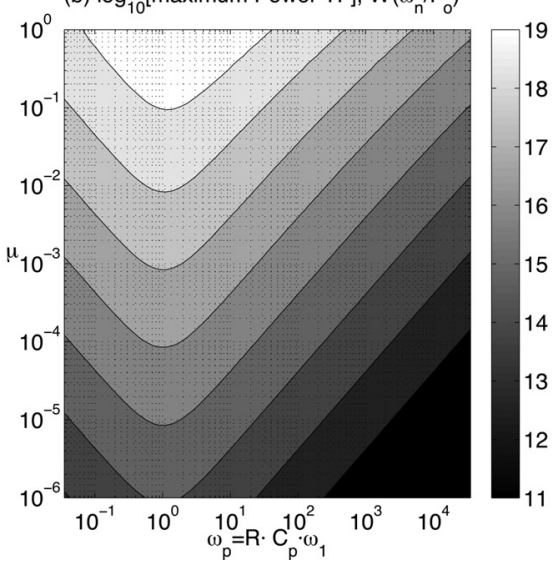

(c) Maximum efficiency

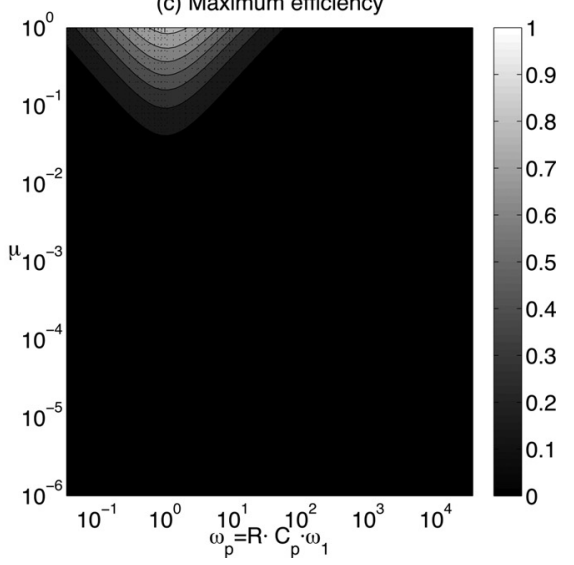

FIG. 9. System responses with weak piezoelectric coupling: (a) main mass vibration attenuation; (b) maximum power TF; (c) maximum efficiency.

efficiency is also constrained to large values of $\mu$. So long as the external circuit is impedance-matched to the choice of the piezoelectric material, $\omega_{p}=1$, the only ceiling to achieving greater power output from the harvesting device is once $\mu \rightarrow 1$. At this limit, the nomenclature of vibration absorber and main dynamic system become irrelevant since the two masses have equal inertial influence.

\section{E. Piezoelectric, strong coupling}

The trends for the performance objectives are slightly modified when considering the EHVA having strong piezoelectric coupling, Fig. 10. The increase in piezoelectric coupling induces greater electrical damping and therefore maximal levels of vibration attenuation. As the mass ratio increases substantially, $\mu \approx 1$, the optimum $\omega_{p}$ begins to shift downward since the primary vibrational frequencies of the system are dramatically affected by the EHVA mass.

The results of maximum power TF show a divergent optimal frequency $\omega_{p}$ as the mass ratio is increased. For large $\mu$, great strain in the piezoelectric spring is induced, leading to larger electromechanical effects. Once the spring strain becomes great enough, around $\mu=0.001$ in Fig. 10(b), tuning of the external circuit away from $\omega_{p}=1$ maintains maximum electrical power. This is a characteristic observed in Refs. 29 and 30 in studies of piezoelectric energy harvesting efficiency. It was found that piezoelectric harvesters with low coupling show maximum power TF for $\omega_{p}=1$. As the coupling strength is increased there appear new choices of $\omega_{p}$ to maximize the electrical power output. This is the result of the piezoelectric stiffening effect observed in the governing equations.

As also observed in Ref. 30, maximum energy harvesting efficiency is still computed to exist at $\omega_{p}=1$ despite the fact that greater electrical power output may be achieved when the circuit is designed for a different $\omega_{p}$ (i.e., uses a different load resistance $R$ ). This indicates that piezoelectric energy harvesting efficiency is not necessarily a metric by which to optimally design the harvester device. The less prominent damping effects and the piezoelectric straincharge coupling itself show that maximum harvesting performance is limited only by a tolerable bound on added mass to the host vibrating structure in solving the simultaneous vibration control problem.

\section{F. Piezoelectric harvesting at resonance}

Figures 11 and 12 plot the piezoelectric oscillator power TF and efficiency computed at the device resonance, $\omega_{2}$. An (a) Vibration attenuation, $\Delta x, d B$

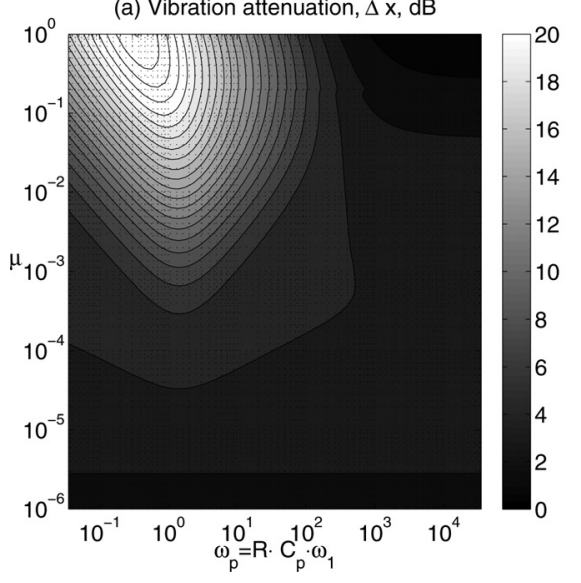

(b) $\log _{10}\left[\right.$ maximum Power TF], W. $\left(\omega_{n}^{2} / F_{0}\right)^{2}$

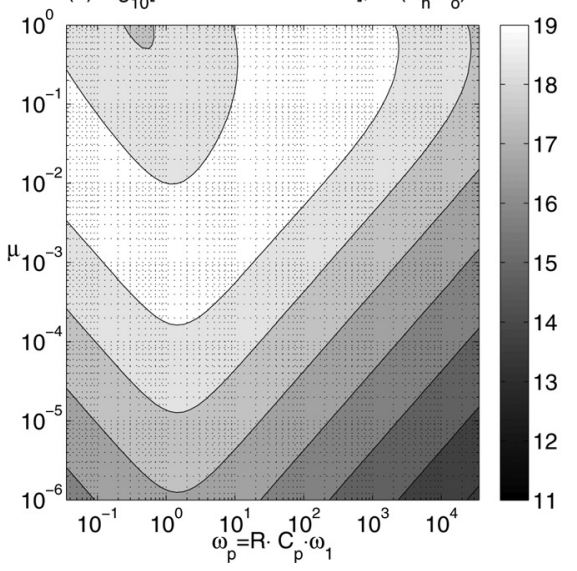

(c) Maximum efficiency

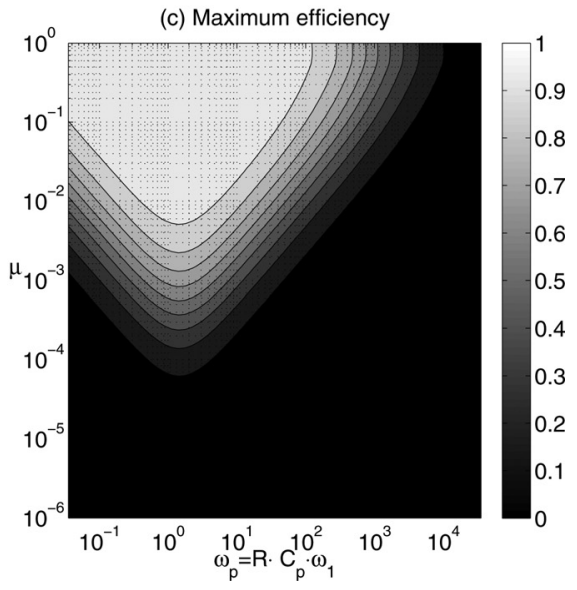

FIG. 10. System responses with strong piezoelectric coupling: (a) main mass vibration attenuation; (b) maximum power TF; (c) maximum efficiency. 
(a) $\log _{10}\left[\right.$ Power TF at $\left.\omega_{2}\right], W \cdot\left(\omega_{n}^{2} / F_{0}\right)^{2}$

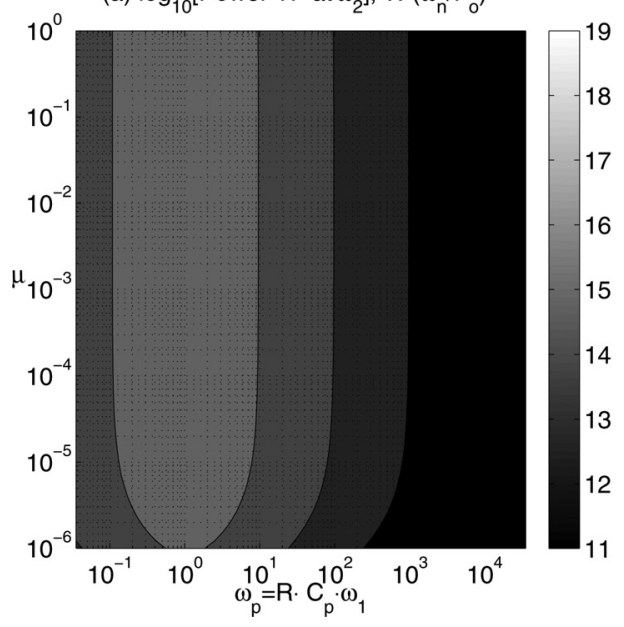

(b) Efficiency at $\omega_{2}$

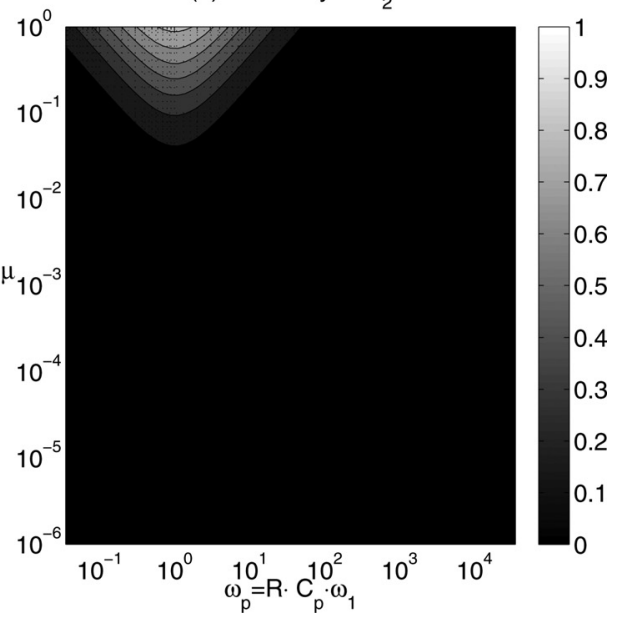

FIG. 11. (a) Power TF of weak piezoelectric oscillator at resonance $\omega_{2}$ and (b) efficiency at resonance. external energy harvesting circuit only capable of switching ac to dc at the piezoelectric device resonance $\omega_{2}$ is substantially less capable of extracting electrical power compared to a circuit able to follow the location of the new split resonances. Figures 11(a) and 12(a) both predict maximum electrical power TF values several orders of magnitude less than those in Figs. 9(b) and 10(b).

This indicates that the coupling mechanism of piezoelectric EHVAs require a robust external harvesting circuit. The explanation follows in steps: since maximum spring deformation is the key to piezoelectric harvesting, a greater mass ratio $\mu$ is desirable to deflect the spring to a greater extent; the larger the oscillator mass, the more dynamic and electromechanical influence the device will have on the total 2DOF system vibration, thus substantially shifting the split resonances from the original oscillator resonance $\omega_{2}$; finally, the external switching circuitry to convert the electrical signal into power will need to compensate for this frequency shift by following one of the split resonances as opposed to "harvesting" at the original tuned frequency $\omega_{2}$.

\section{CONCLUDING REMARKS}

The fundamental, dimensionless governing equations of an excited main mass-spring-damper to which either an electromagnetic or piezoelectric energy harvesting vibration absorber is attached were derived. This represents the extension of classical dynamic vibration absorber analysis to include passive electromechanical damping and stiffening effects with the additional objective function of maximizing harvested electrical power.

Electromagnetic EHVAs achieve greater induced damping for light weight oscillator masses. Thus, compared with piezoelectric EHVAs, electromagnetic devices may suppress the host mass vibration to a greater degree with less added mass. This is evident in the governing equations since the coupling coefficients for electromagnetic EHVAs are roughly on the same order whereas piezoelectric coupling terms show many orders of magnitude in difference.

Electromagnetic energy harvesters maximize electrical output by increasing the cyclic displacement stroke of the oscillating mass while piezoelectric harvesters maximize electrical output by increasing the magnitude of spring deformation. Therefore, it is found that electromagnetic EHVAs achieve a maximum power TF at the device's original resonance, $\omega_{2}$. The strength of the electromagnetic coupling serves to shift the optimum choice of device mass ratio, $\mu$, for maximum electrical power output and harvesting efficiency such that no split resonances are induced in the coupled system.

\section{(a) $\log _{10}\left[\right.$ Power TF at $\left.\omega_{2}\right], W \cdot\left(\omega_{n}^{2} / F_{0}\right)^{2}$}

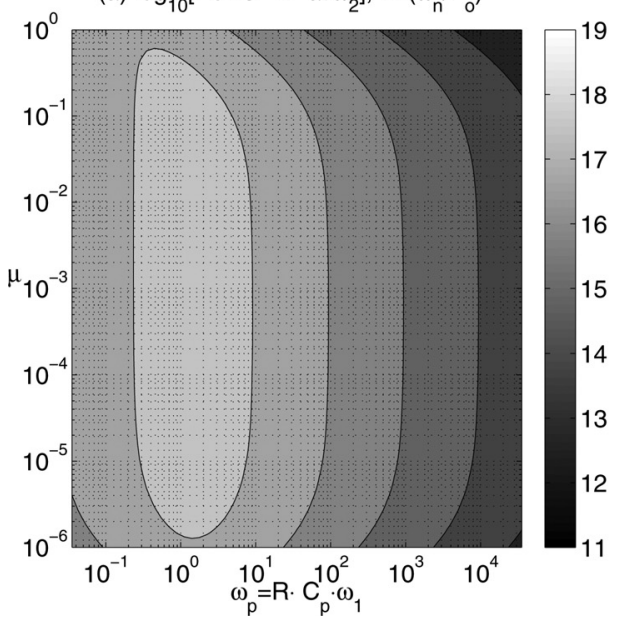

(b) Efficiency at $\omega_{2}$

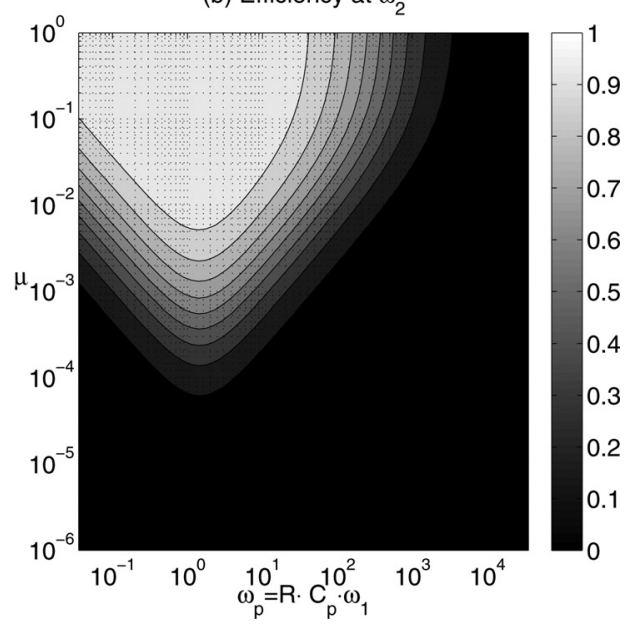

FIG. 12. (a) Power TF of strong piezoelectric oscillator at resonance $\omega_{2}$ and (b) efficiency at resonance. 
Piezoelectric EHVAs exhibit different characteristics due to their electromechanical conversion mechanism. Heavier oscillators, which more greatly deform the piezoelectric spring, are the best choice for energy harvesting objectives, to the point at which the oscillator is on the same order of mass as the host mass. The strength of piezoelectric coupling highly influences the design of the harvesting circuitry, i.e., the realistic ac-dc switching circuit, since the variable piezoelectric stiffening and damping effects shift the operating conditions for maximum electrical power away from the original EHVA resonance, $\omega_{2}$. This is in addition to the production of split system resonances as the harvester becomes more massive. Therefore, optimized piezoelectric EHVAs must carefully adjust more design parameters for harvesting objectives than optimized electromagnetic EHVAs.

APPENDIX A: CLOSED FORM SOLUTION TO EQ. (4)

$$
\begin{aligned}
& x(r)=\frac{F_{o} / \omega_{1}^{2}}{1+\mu \Theta^{2}-r^{2}+j 2 r\left(\zeta_{1}+\zeta_{1} \mu \Theta\right)-\frac{\left(\mu \Theta^{2}+j 2 \zeta_{2} \mu \Theta r\right)^{2}}{\mu \Theta^{2}-\mu r^{2}+j 2 \zeta_{2} \mu \Theta r+\frac{r}{L}\left(\frac{T}{\omega_{1}}\right)^{2} /\left(r-j \omega_{e}\right)}}, \\
& y(r)=\frac{\mu \Theta^{2}+j 2 \zeta_{2} \mu \Theta r}{\mu \Theta^{2}-\mu r^{2}+j 2 \zeta_{2} \mu \Theta r+\frac{r}{L}\left(\frac{T}{\omega_{1}}\right)^{2} /\left(r-j \omega_{e}\right)} x(r), \\
& q(r)=j\left[\left(\frac{T}{\omega_{1} L}\right) /\left(r-j \omega_{e}\right)\right] y(r) .
\end{aligned}
$$

\section{APPENDIX B: CLOSED FORM SOLUTION TO EQ. (6)}

$$
x(r)=\frac{\left(F_{o} / \omega_{1}^{2}\right)\left[1-\left(\frac{\Theta}{r}\right)^{2}-j 2 \zeta_{2} \frac{\Theta}{r}-j \alpha(r)\right]}{1+\mu \Theta^{2}-r^{2}+j 2 r\left(\zeta_{1}+\zeta_{2} \mu \Theta\right)+j \alpha(r) \mu r^{2}-\left\{\left[\left(\frac{\Theta}{r}\right)^{2}+j 2 \zeta_{2} \frac{\Theta}{r}\right]+j \alpha(r)\right\}\left[1-r^{2}+j 2 \zeta_{1} r\right]},
$$

$$
\begin{aligned}
& y(r)=\frac{\left(1-r^{2}+j 2 \zeta_{1} r\right) x(r)-F_{o} / \omega_{1}^{2}}{\mu r^{2}}, \\
& v(r)=-j \frac{\alpha(r) r^{2}}{d_{33} \Theta^{2}} x(r)+j \frac{\alpha(r) r^{2}}{d_{33} \Theta^{2}} y(r), \\
& \alpha(r)=\frac{d_{33}^{2} \omega_{1}^{2} \mu \Theta^{4}}{C_{p}\left[\frac{r}{\omega_{p}}+j r^{2}\left(1-k^{2}\right)\right]} .
\end{aligned}
$$

${ }^{1}$ N. E. duToit, B. L. Wardle, and S. -G. Kim, "Design considerations for mems-scale piezoelectric mechanical vibration energy harvesters," Integr. Ferroelectr. 71, 121-160 (2005).

${ }^{2}$ S. P. Beeby, M. J. Tudor, and N. M. White, "Energy harvesting vibration sources for microsystems applications," Meas. Sci. Technol. 17, R175-R195 (2006).

${ }^{3}$ K. A. Cook-Chennault, N. Thambi, and A. M. Sastry, "Powering MEMS portable devices-A review of non-regenerative and regenerative power supply systems with special emphasis on piezoelectric energy harvesting systems," Smart Mater. Struct. 17(4), 043001 (2008).

${ }^{4}$ H. Sodano, G. Park, and D. J. Inman, "A review of power harvesting from vibration using piezoelectric materials," Shock Vib. Dig. 36, 197-205 (2004).

\footnotetext{
${ }^{5}$ S. Priya, "Advances in energy harvesting using low profile piezoelectric transducers," J. Electroceram. 19, 167-184 (2007).

${ }^{6}$ N. E. duToit and B. L. Wardle, "Performance of microfabricated piezoelectric vibration energy harvesters," Integr. Ferroelectr. 83(1), 13-32 (2006).

${ }^{7}$ A. Erturk and D. J. Inman, "An experimentally validated bimorph cantilever model for piezoelectric energy harvesting from base excitations," Smart Mater. Struct. 18(2), 025009 (2009).

${ }^{8}$ M. A. Karami and D. J. Inman, "Equivalent damping and frequency change for linear and nonlinear hybrid vibrational energy harvesting systems," J. Sound Vib. 330(23), 5583-5597 (2011).

${ }^{9}$ N. G. Stephen, "On energy harvesting from ambient vibration," J. Sound Vib. 293, 409-425 (2006).

${ }^{10}$ G. Poulin, E. Sarraute, and F. Costa, "Generation of electrical energy for portable devices comparative study of an electromagnetic and piezoelectric system," Sens. Actuators, A 116, 461-471 (2004).

${ }^{11}$ A. Erturk, "Piezoelectric energy harvesting for civil infrastructure system applications: Moving loads and surface strain fluctuations," J. Intell. Mater. Syst. Struct. 22(17), 1959-1973 (2011).

${ }^{12}$ Y. Tadesse, S. Zhang, and S. Priya, "Multimodal energy harvesting system: Piezoelectric and electromagnetic," J. Intell. Mater. Syst. Struct. 20, 625-632 (2009).

${ }^{13}$ J. P. Den Hartog, Mechanical Vibrations, 4th ed. (Dover, New York, 1985), pp. 87-104.
} 
${ }^{14}$ I. L. Cassidy, J. T. Scruggs, S. Behrens, and H. P. Gavin, "Design and experimental characterization of an electromagnetic transducer for largescale vibratory energy harvesting applications," J. Intell. Mater. Syst. Struct. 22(17), 2009-2024 (2011).

${ }^{15} \mathrm{~T}$. Ni, L. Zuo, and A. Kareem, "Assessment of energy potential and vibration mitigation of regenerative tuned mass dampers on wind excited tall buildings," in ASME 2011 International Design Engineering Technical Conferences \& Computers and Information in Engineering Conference, Washington, DC (2011).

${ }^{16} \mathrm{X}$. Tang and L. Zuo, "Simulation and experimental validation of simultaneous vibration control and energy harvesting from buildings using tuned mass dampers," in 2011 American Control Conference, San Francisco (2011).

${ }^{17}$ M. Ouled Chtiba, S. Choura, A. H. Nayfeh, and S. El-Borgi, "Vibration confinement and energy harvesting in flexible structures using collocated absorbers and piezoelectric devices," J. Sound Vib. 329, 261-276 (2010).

${ }^{18}$ R. L. Harne, "Concurrent attenuation of and energy harvesting from surface vibrations: Experimental verification and model validation," Smart Mater. Struct. 21, 035016 (2012).

${ }^{19}$ Y. -T. Choi and N. M. Wereley, "Self-powered magnetorheological dampers," J. Vib. Acoust. 131, 044501 (2009).

${ }^{20}$ L. Zuo and P. -S. Zhang, "Energy harvesting, ride comfort, and road handling of regenerative vehicle suspensions," ASME Conf. Proc. 2011, 295 (2011).

${ }^{21}$ J. W. Xu, W. W. Shao, F. R. Kong, and Z. H. Feng, "Right-angle piezoelectric cantilever with improved energy harvesting efficiency," Appl. Phys. Lett. 96, 152904 (2010).
${ }^{22}$ W. Zhou, G.R. Penamalli, and L. Zuo, "An efficient vibration energy harvester with a multi-mode dynamic magnifier," Smart Mater. Struct. 21, 015014 (2012).

${ }^{23}$ M. Arafa, W. Akl, A. Aladwani, O. Aldraihem, and A. Baz, "Experimental implementation of a cantilevered piezoelectric energy harvester with dynamic magnifier," Proc. SPIE 7977, 79770Q (2011).

${ }^{24} \mathrm{X}$. Tang and L. Zuo, "Enhanced vibration energy harvesting using dualmass systems," J. Sound Vib. 330(21), 5199-5209 (2011).

${ }^{25}$ J. J. McCoy, "Harvesting mechanical energy via structural vibrations," J. Acoust. Soc. Am. 130(4), 1783-1786 (2011).

${ }^{26}$ A. Preumont, Mechatronics: Dynamics of Electromechanical and Piezoelectric Systems (Springer, Berlin, 2006).

${ }^{27}$ L. Cremer, M. Heckl, and B. A. T. Petersson, Structure-Borne Sound: Structural Vibrations and Sound Radiation at Audio Frequencies, 3rd ed. (Springer, Berlin, 2005), Chap. 4.

${ }^{28}$ C. D. Richards, M. J. Anderson, D. F. Bahr, and R. F. Richards, "Efficiency of energy conversion for devices containing a piezoelectric component," J. Micromech. Microeng. 14, 717-721 (2004).

${ }^{29}$ Y. C. Shu and I. C. Lien, "Efficiency of energy conversion for a piezoelectric power harvesting system," J. Micromech. Microeng. 16, 2429-2438 (2006).

${ }^{30}$ Y. Liao and H. A. Sodano, "Structural effects and energy conversion efficiency of power harvesting," J. Intell. Mater. Syst. Struct. 20, 505-514 (2009).

${ }^{31}$ T. Asami, O. Nishihara, and A. M. Baz, "Analytical solutions to $h_{\infty}$ and $h_{2}$ optimization of dynamic vibration absorbers attached to damped linear systems," J. Vib. Acoust. 124, 284-295 (2002).

${ }^{32}$ L. Zuo and S. A. Nayfeh, "Optimization of the individual stiffness and damping parameters in multiple-tuned-mass-damper systems," J. Vib. Acoust. 127, 77-83 (2005). 